\title{
THE CAROTID SHUDDER
}

\author{
BY \\ WILLIAM EVANS AND DAVID LEWES \\ From the Cardiac Department of the London Hospital \\ Received August 28, 1945
}

The diagnosis of aortic stenosis in the presence of aortic incompetence needs to be strengthened. A rough systolic murmur in the aortic area is not always accompanied by a thrill. Again, a thrill in the neck cannot be accepted as evidence of aortic stenosis without other supporting signs, especially if the thrill is short and the subject young when a systolic murmur is not expected in the aortic area. In nine patients in whom subsequent clinical, cardiographic, and cardioscopic examination, established the presence of aortic stenosis and incompetence, we observed a characteristic sign, which by itself permitted a diagnosis of the combined aortic lesion from inspection of the neck. At the height of the carotid pulse, large on account of aortic incompetence, we noticed a quiver or vibration which lasted for a moment only. This effect we have named the carotid shudder for it best describes the visual clinical sign.

We are not aware that it has been described before, but recently we have read in Paul White's book on Heart Disease (1931) a statement that one of his assistants had drawn his attention to a curious vibration in the neck of a young patient with aortic stenosis; there was no mention of associated aortic incompetence, and although it was implied that it was unique and not distinctive for any group of patients, it is probable that the phenomenon was the same as the one discussed here.

The sign was recorded by placing a tambour over the carotid pulsation and connecting it with a Mackenzie's polygraph. Records obtained in this way were examined alongside those obtained in healthy subjects and in patients with lone aortic incompetence or with hypertension producing a kinked carotid. The tracing in carotid shudder, of which that shown in Fig. 1 is representative, was taller than in health, but no taller than the one obtained in

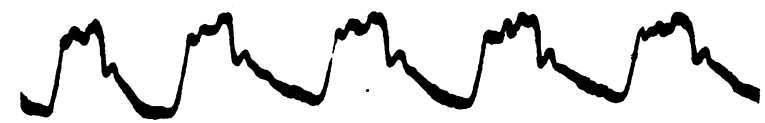

FIG. 1.-Carotid arteriogram in a patient with aortic incompetence and stenosis in whom carotid shudder was observed.

lone aortic incompetence or over a kinked carotid, although it was usually broader and showed characteristic serrations from the vibration or shudder effect. Such vibration usually occurred at the height of the upstroke. None of the control cases showed all three features of the carotid shudder curve, namely tall, broad, and serrated. The tracings, however, failed to portray adequately the characteristic vibration which was so distinctive on clinical inspection of the carotid pulse.

Only a proportion of patients with conjoined aortic stenosis and aortic incompetence exhibit carotid shudder, but the fact that we were able to collect nine such cases during eighteen months emphasizes its common incidence. The clinical sign was sought in patients where aortic incompetence or aortic stenosis existed separately, but it was not found once.

Clinical examination. On further examination of the nine patients the pulse was often collapsing in character with a raised pulse pressure commensurate with the degree of aortic incompetence accompanying the stenosis. Pulsation was often obvious in the suprasternal notch, but never as prominent as in the carotid arteries. There was always a long systolic 
thrill in the neck which was felt best above the right clavicle; in six cases the thrill was elicited in the aortic area as well. The apex beat was displaced outwards, and loud systolic and early diastolic murmurs could be heard between the aortic and mitral areas. The ætiology was rheumatic in eight patients and luetic in one.

Electrocardiogram. This was abnormal as a rule, and the changes included inversion of the $\mathrm{T}$ wave, depression of the R-T segment, and left electrical axis deviation. The signs of left ventricular preponderance were often present and consisted of left axis deviation, inversion of the $T$ wave in lead $I$, and greater inversion of the $T$ wave in lead $C_{7}$ than in IVR.

Cardioscopy. The findings included enlargement of the left ventricle in proportion to the degree of incompetence present, outward curving of the ascending aorta, and extension of the left limb of the arc, which formed the aortic knuckle, towards the left clavicle; increased aortic pulsation produced an upward projection of this limb during systole, and displaced the main arc to the right. When viewed in the left oblique position the aortic elongation did not always raise the aortic arch as in hypertension, but it sometimes displaced the limbs of the arch apart and widened the base of the aortic triangle above (Fig. 2). In other cases

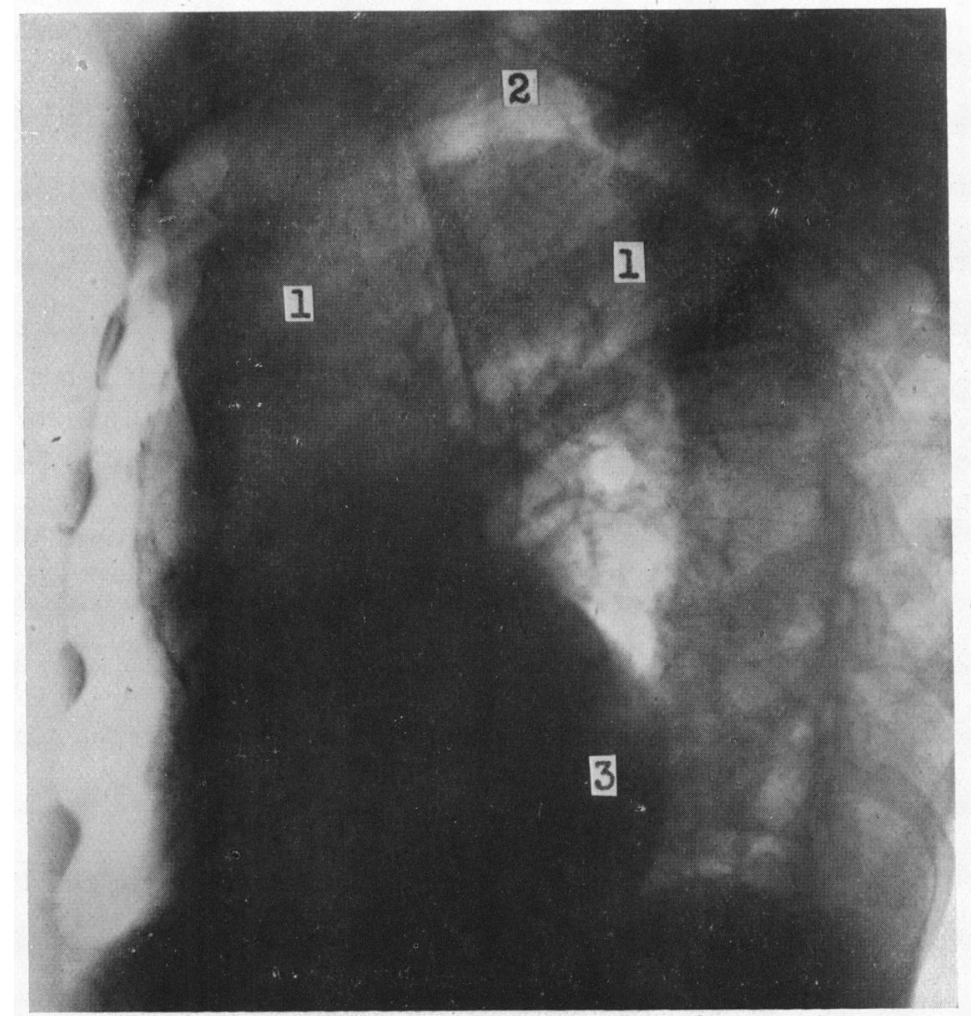

Fig. 2.-Left oblique (II) view in a male, aged 58, with aortic incompetence and stenosis, who showed carotid shudder. Elongation without elevation of the aortic arch (1) has widened the base of the aortic triangle (2). Enlargement of the left ventricle (3) is shown.

the aortic arch was neither elongated nor raised. Such effects show that the mechanism of carotid shudder is not related to any change in the alignment of the artery, but probably depends on an increase in the volume of blood discharged into the vessel with each systole and the impedient effect of the stenosed aortic valve on the returning flow to the left ventricle.

\section{CONCLUSION}

When the increased carotid pulse exhibits vibration at the height of the systolic excursion, it signifies that aortic incompetence and aortic stenosis exist side by side. We have named this sign the carotid shudder, and although it is not found in all cases of aortic incompetence and stenosis, its presence is a sure indication of the dual lesion. 